\title{
Role of the Angular Distribution of Backscattered Electrons in Low Energy Scanning Electron Microscope
}

\author{
Ilona Müllerová1, Luděk Frank, Ivo Konvalina, and Eliška Mikmeková \\ ${ }^{1}$ Department of Electron Microscopy, Institute of Scientific Instruments ASCR, v.v.i., Brno, Czech \\ Republic.
}

The single-channel detection systems are the most often used for the detection of the Secondary Electrons (SE) and Backscattered Electrons (BSE) in the Scanning Electron Microscope (SEM). Sometimes the segmented detector of BSE according to the azimuthal angle is used to optimize the material (sum signal) and topographic contrast (subtracted signal). To achieve high lateral resolution the specimen is usually immersed in the strong magnetic field and/or in the strong electrostatic field, in the case of the operation at low primary beam energies; we call this regime Cathode Lens Mode (CLM) [1]. Those fields strongly influence the trajectories of the signal electrons [2].

Figure 1a shows straight trajectories of the simulated signal electrons emitted from the specimen under polar angle from $0 \mathrm{deg}$ to $910 \mathrm{deg}$ with respect to the optical axis with the step of $10 \mathrm{deg}$. Figure $1 \mathrm{~b}$ shows the same trajectories but the specimen in immersed in the electrostatic field, in this case. The electrostatic field between the specimen at negative potential and the detector on the ground potential is $450 \mathrm{~V} / \mathrm{mm}$, distance between the specimen and the detector is about $9 \mathrm{~mm}$. You can see big contrast between the precipitates and the substrate in the Figure 1d, even though the difference in the atomic number is only 0.14 , because the electrons emitted under large angle with respect to the optical axis are collected. We cannot see such a big contrast in the standard system (Figure 1c) even though the landing energy of the primary beam electrons is the same in both cases.

We have designed a multi-channel detector collecting BSE electrons to learn more about the angular distribution of the reflected electrons (Figure 2). The detector consists of a grid on the ground potential, followed by the multi-channel plate (MCP) and the eight-channel collector at the ground potential (Figure 3). The active area of each segment is $32 \mathrm{~mm}^{2}$. The collimation of the signal trajectories toward the optical axis can be controlled via the immersion ratio $\mathrm{k}=\mathrm{E}_{\mathrm{P}} / \mathrm{E}_{\mathrm{L}}$ (primary beam energy of electrons / landing energy of electrons, which is controlled by the negative potential on the specimen).

The microstructure was imaged by this segmented detector at $5 \mathrm{keV}$ of landing energy. Figure 4 right shows the structure imaged by the individual channels when no field is applied to the specimen region, when only small part of the emitted electrons is collected. Strong contrast changes appear when nearly all electrons emitted from the specimen are collected by the individual segments. [3].

\section{References:}

[1] I Müllerová and L Frank, Advances in Imaging and Electron Physics 128 (2003), p. 309.

[2] I. Konvalina and I. Müllerová, Nuclear Instr. \& Methods in Phys. Res. Sec. A 645, 1 (2011), p. 55.

[3] The authors acknowledge funding from the Technology Agency of the Czech Republic (Competence center Electron microscopy, no: TE01020118) and from the People Programme (Marie Curie Actions) of the European Union's Seventh Framework Programme FP7/2007-2013/ under REA grant agreement no: 606988. 

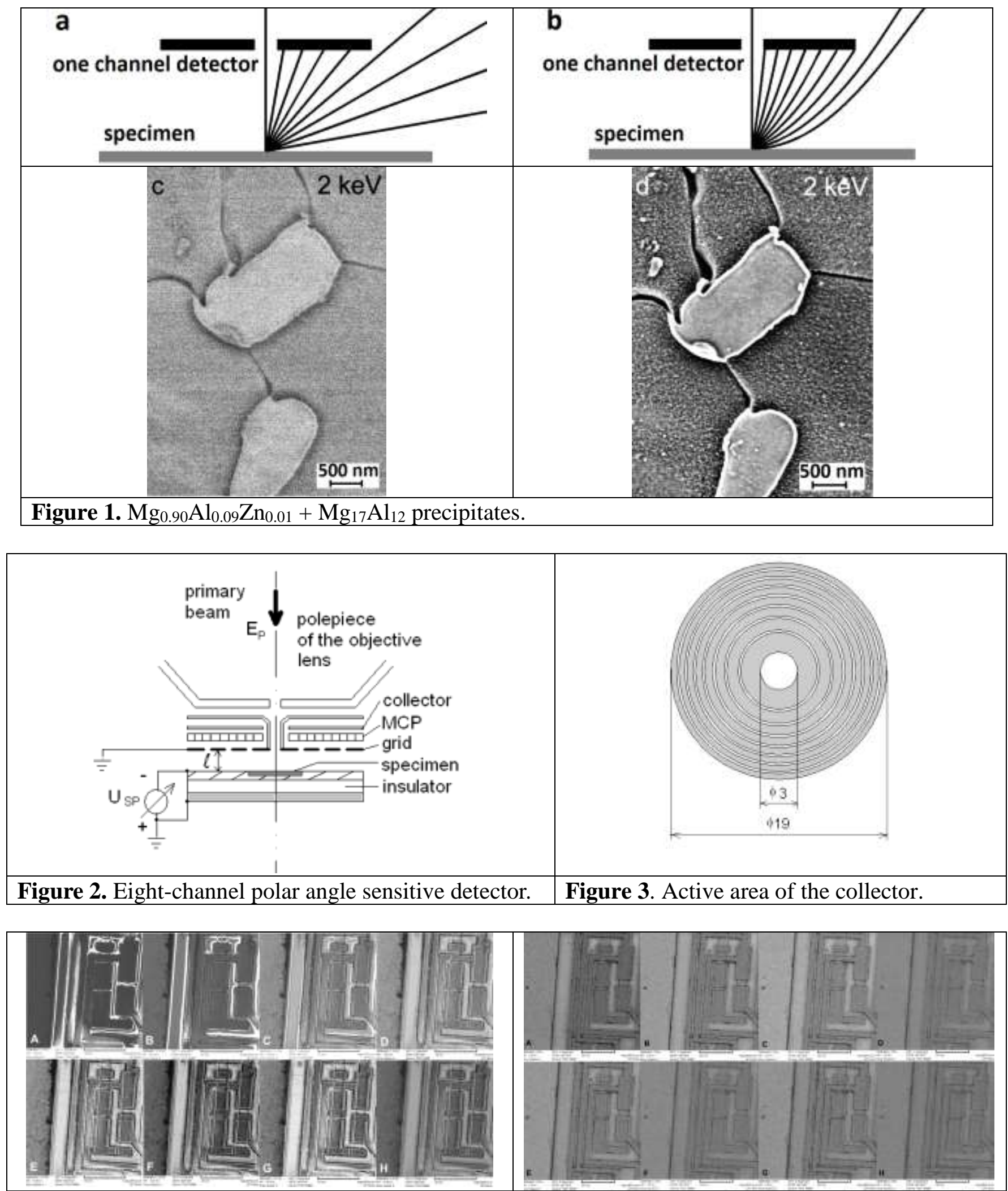

Figure 4. Microstructure imaged by eight-channel detector at $5 \mathrm{keV}$. Cathode lens mode (left), standard mode (right). 